\title{
Analysis of an age-structured predator-prey model with disease in the prey ${ }^{\star}$
}

\author{
Manuel Delgado, Mónica Molina-Becerra ${ }^{1, *}$ and Antonio Suárez \\ Dpto Ecuaciones Diferenciales y Análisis Numérico, Universidad de Sevilla, Apdo \\ de Correos 1160, 41080 Sevilla, Spain
}

\begin{abstract}
We study a nonlinear predator-prey model in which the prey population is affected by a mild disease, but has an effect on the death-rate and is assumed to have age structure. We assume that the predator population grows according to a logistic law. We prove the existence and uniqueness of a positive solution for this model.
\end{abstract}

Key words: Age-structure, predator-prey model, integro-differential equations decoupling

\section{Introduction}

Population dynamic models have been developed by the consideration of the age-dependent structure. A simple age-dependent population dynamics model was first proposed by Lotka and Von Foerster $[1,2]$. Its main disadvantage is that the birth and the death processes are independent of the total population size. In their pioneering work, Gurtin and MacCamy [3], to overcome this deficiency, considered a nonlinear age-dependent populations model, whose the birth and the death processes depend on the total population (see the book by Webb [4] for a survey).

* This work has been partially supported by BFM 2000-0797.

* Corresponding author. Fax:(34) 954552898

Email addresses: delgado@numer.us.es (Manuel Delgado), monica@numer.us.es (Mónica Molina-Becerra), suarez@numer.us.es (Antonio Suárez).

1 Research financed by Patronato de la Fundación Cámara de la Universidad de Sevilla. 
It was later studied the dynamic of a population divided in two interacting subpopulations where the parameters such as fecundity, mortality and interaction coefficients are assumed to be age-dependent. This has been the basis of the study of epidemic models (see, [5-7] for instance).

Predator-prey models with disease in the prey have been recently studied (see [8] for example) but the authors do not consider any structure in age.

In this work, we consider a system involving two species, a prey $(p)$ and a predator $(Y)$. The prey population is affected by a mild disease, in the sense that it allows the partial recuperation of infected individuals and is structured in age. We assume that the infection weakens the prey and increases its susceptibility to predation, whereas the predator is not affected by the disease. From now on, we suppose the following assumptions:

In the absence of the prey population, the predator grows according to a logistic law, then

$$
\dot{Y}=m Y(1-Y / D),
$$

where $m$ and $D$ are positive constants. The constant $D$ is the carrying capacity of the environment, which is usually determined by the available sustaining resources. The constant $m$ is an intrinsic birth rate of predator. For simplicity of notation, we write $n$ instead of $m / D$.

In the absence of the predator population, we consider that a contagious phenomenon acts on the prey. This leads to a population divided into susceptible and infective individuals. The age-specific densities of the susceptible, infective and prey population at time $t$ and age $a$ are denoted by $s(a, t), i(a, t)$ and $p(a, t)$, respectively. It is clear that $p(a, t)=i(a, t)+s(a, t)$.

The age-specific force of infection that we consider has been previously used in [9]. Namely,

$$
\lambda(a, t ; i)=\theta(a) i(a, t)+\int_{0}^{\infty} K\left(a, a^{\prime}\right) i\left(a^{\prime}, t\right) d a^{\prime},
$$

where $K\left(a, a^{\prime}\right)$ is the rate at which an infective individual of age $a^{\prime}$ comes into a disease transmitting contact with a susceptible individual of age $a$ and $\theta(a)$ denote the infection rate for pure intracohort case.

We will write $P$ for the size of total population, i.e. $P(t)=\int_{0}^{\infty} p(a, t) d a$, and $\mu_{1}(a, t, P(t)), \mu_{2}(a, t, P(t))$ the age-specific mortality of the infective and of the susceptible individuals at time $t$ respectively. We assume the disease affecting the death rate, so we have that $\mu_{1}(a, t, P(t)) \geq \mu_{2}(a, t, P(t))$. We would like to point out that so far it was studied in the case $\mu_{1}=\mu_{2}$, for which an specific change of variables works (see [6,7,9] for instance). We improve these results studying the case $\mu_{1} \neq \mu_{2}$. 
We write $\beta$ for the birth rate and we assume that the birth is modelled by

$$
\begin{aligned}
i(0, t) & =\int_{0}^{\infty} q \beta(a, t, P(t)) i(a, t) d a \\
s(0, t) & =\int_{0}^{\infty} \beta(a, t, P(t))(s(a, t)+(1-q) i(a, t)) d a
\end{aligned}
$$

for $t>0$ and where $q \in[0,1]$ is the vertical transmission, that is, the ratio of infective newborns produced by infective. Hence we have that all offspring of susceptible parents are susceptible.

We also suppose that the initial age distributions are given by $s_{0}, i_{0}$. If we write by $\gamma$ the age-specific recovery rate, we obtain that the dynamic of the prey in absence of predator is governed by the following system of equations

$$
\left\{\begin{array}{l}
\frac{\partial i}{\partial t}+\frac{\partial i}{\partial a}+\mu_{1}(a, t, P(t)) i(a, t)=\lambda(a, t ; i) s(a, t)-\gamma(a) i(a, t), \\
\frac{\partial s}{\partial t}+\frac{\partial s}{\partial a}+\mu_{2}(a, t, P(t)) s(a, t)=-\lambda(a, t ; i) s(a, t)+\gamma(a) i(a, t), \\
i(a, 0)=i_{0}(a), s(a, 0)=s_{0}(a), \\
i(0, t)=q \int_{0}^{\infty} \beta(a, t, P(t)) i(a, t) d a \\
s(0, t)=\int_{0}^{\infty} \beta(a, t, P(t))(s(a, t)+(1-q) i(a, t)) d a .
\end{array}\right.
$$

It is logical to suppose that $i(a, t), s(a, t) \longrightarrow 0$, when $a \rightarrow+\infty$.

In the presence of the prey and the predator, we denote by $\varepsilon>0$ the coefficient in converting prey into predator, the predation rate on infected and on susceptible prey by the constants $M_{1}>0$ and $N_{1}>0$, respectively. Since we consider the case when the predator mainly eats the infected prey, we can assume that $M_{1} \geq M_{2}$.

Taking all the above into account, we have to study the following model

$$
\left\{\begin{array}{l}
\frac{\partial i}{\partial t}+\frac{\partial i}{\partial a}+\mu_{1}(a, t, P(t)) i=\lambda(a, t ; i) s-\gamma(a) i(a, t)-M_{1} i(a, t) Y(t), \\
\frac{\partial s}{\partial t}+\frac{\partial s}{\partial a}+\mu_{2}(a, t, P(t)) s=-\lambda(a, t ; i) s+\gamma(a) i(a, t)-M_{2} s(a, t) Y(t), \\
\dot{Y}=m Y(t)-n Y^{2}(t)+\varepsilon M_{1} I(t) Y(t)+\varepsilon M_{2} S(t) Y(t), \\
i(a, 0)=i_{0}(a), s(a, 0)=s_{0}(a), Y(0)=Y_{0}, \\
i(0, t)=q \int_{0}^{\infty} \beta(a, t, P(t)) i(a, t) d a, \\
s(0, t)=\int_{0}^{\infty} \beta(a, t, P(t))(s(a, t)+(1-q) i(a, t)) d a, \\
i(a, t), s(a, t) \longrightarrow 0, \text { when } a \rightarrow \infty,
\end{array}\right.
$$

In [10], an age-structured population model with $N$ class of the population is studied under the hypotheses of linear rates mortality and lipschitzianity 
of the interaction coefficients. In that paper, it is used integrated solutions of the problem. This method is not applicable to our model.

In this paper, we prove the existence and uniqueness of nonnegative solution of the model (3) on any finite time-interval which has nonlinear rate mortality and the age-dependent force of infection term is not lipschtzian. Our results are based in a process of decoupling of the age-dependent problem for the prey and the predator and later a fixed point method.

An outline of this work is as follows: Section 2 establishes the relation between the system (3) and the problem that involves only the prey population and a Bernoulli's o.d.e. In the Section 3, we proceed with the study of existence and uniqueness of a solution for an epidemic model with different mortality rates. To do that, we need the following hypothesis. Given $T>0$, we denote $I:=[0, T]$ and we suppose that

(H1) For $i=1,2, \mu_{i}(a, t, P)$ is a nonnegative measurable function such that the mapping $s \longmapsto \mu_{i}(s, s+u, P)$ belongs to $L_{L o c}^{1}\left(\mathbb{R}_{+}\right)$for almost all $(u, P) \in \mathbb{R}^{2}$. And there exists a constant $C(T)>0$ such that for all $P, P^{\prime} \in \mathbb{R}$

$$
\left|\mu_{i}(a, t, P)-\mu_{i}\left(a, t, P^{\prime}\right)\right| \leq C(T)\left|P-P^{\prime}\right| \text { a.e. }(a, t) \in \mathbb{R}_{+} \times I .
$$

With the notation $\mu=\mu_{1}-\mu_{2}$, there exists another constant $C(T)>0$ such that

$$
|\mu(a, t, P)| \leq C(T) \log (|P|+e) \text { a.e. }(a, t) \in \mathbb{R}_{+} \times I .
$$

(H2) $\beta(a, t, P)$ is a nonnegative measurable function which has compact support on the variable $a$ and such that for all $P, P^{\prime} \in \mathbb{R}$

$$
\left|\beta(a, t, P)-\beta\left(a, t, P^{\prime}\right)\right| \leq C(T)\left|P-P^{\prime}\right| \text { a.e. }(a, t) \in \mathbb{R}_{+} \times I
$$

where $C(T)>0$ is another constant which depends only on $T$. Moreover, there exists a constant $C(T)>0$ such that for all $P \in \mathbb{R}$

$$
|\beta(a, t, P)| \leq C(T) \log (|P|+e) \text { a.e. }(a, t) \in \mathbb{R}_{+} \times I .
$$

We would like to point that the estimates (5) and (7) were motivated by $[11]$.

(H3) $\psi_{0}:=\left(i_{0}, s_{0}\right) \in\left(L^{1}\left(\mathbb{R}_{+}\right)\right)^{2}$ has compact support.

(H4) $\theta, \gamma \in L^{\infty}\left(\mathbb{R}_{+}\right)$have compact support and are nonnegative functions. We denote by $\theta_{\infty}=\operatorname{dess} \sup _{a \in(0, \infty)} \theta(a)$ and $\gamma_{\infty}=\operatorname{dess} \sup _{a \in(0, \infty)} \gamma(a)$

(H5) $K \in L^{\infty}\left(\mathbb{R}_{+} \times \mathbb{R}_{+}\right)$has compact support and is a nonnegative function. We denote by $K_{\infty}=\operatorname{dess} \sup _{a \in(0, \infty), a^{\prime} \in(0, \infty)} K\left(a, a^{\prime}\right)$

Finally, Section 4 is devoted to study the o.d.e. by means of a fixed point argument. 
In future works, we try to determine asymptotic behavior of the system (3) and we shall discuss some examples.

\section{Reduction of the model}

In this Section, we will see that the study of existence and uniqueness properties of the model (3) is equivalent to analyze the model (2) and a certain o.d.e.

Let $T>0$, and $z \in \mathcal{C}\left(I ; \mathbb{R}_{+}\right)$be, we denote

$$
\mu_{i, z}(a, t, P):=\mu_{i}(a, t, P)+M_{i} z(t), i=1,2
$$

For each $z \in \mathcal{C}\left(I ; \mathbb{R}_{+}\right)$, we consider the problem

$$
\left\{\begin{array}{l}
\frac{\partial i_{z}}{\partial t}+\frac{\partial i_{z}}{\partial a}+\mu_{1, z}\left(a, t, P_{z}(t)\right) i_{z}=\lambda\left(a, t ; i_{z}\right) s_{z}-\gamma(a) i_{z}(a, t) \\
\frac{\partial s_{z}}{\partial t}+\frac{\partial s_{z}}{\partial a}+\mu_{2, z}\left(a, t, P_{z}(t)\right) s_{z}=-\lambda\left(a, t ; i_{z}\right) s_{z}+\gamma(a) i_{z}(a, t) \\
i_{z}(a, 0)=i_{0}(a), \quad s_{z}(a, 0)=s_{0}(a), \\
i_{z}(0, t)=q \int_{0}^{\infty} \beta\left(a, t, P_{z}(t)\right) i_{z}(a, t) d a \\
s_{z}(0, t)=\int_{0}^{\infty} \beta\left(a, t, P_{z}(t)\right)\left(s_{z}(a, t)+(1-q) i_{z}(a, t)\right) d a \\
i_{z}(a, t), s_{z}(a, t) \longrightarrow 0, \text { when } a \rightarrow \infty
\end{array}\right.
$$

where $P_{z}=I_{z}+S_{z}:=\int_{0}^{\infty} i_{z}(a, t) d a+\int_{0}^{\infty} s_{z}(a, t) d a$.

For each $z \in \mathcal{C}\left(I ; \mathbb{R}_{+}\right)$, let us suppose that this system has a unique nonnegative solution $\left(i_{z}, s_{z}\right) \in L^{\infty}\left(I ;\left(L^{1}\left(\mathbb{R}_{+}\right)\right)^{2}\right)$. Then, to prove that the model (3) has a unique solution is sufficient to study the o.d.e.

$$
\frac{d w}{d t}(t)=m w(t)-n w^{2}(t)+M_{1} P_{z}(t) w(t)+\varepsilon\left(M_{2}-M_{1}\right) S_{z}(t) w(t)
$$

with $w(0)=Y_{0}$.

Observe that (10) is a Bernoulli's o.d.e. and we can solve it explicitly

$$
w(t)=\frac{Y_{0} e^{m t} \exp \left[f_{z}(t)\right]}{1+n Y_{0} \int_{0}^{t} e^{m \tau} \exp \left[f_{z}(\tau)\right] d \tau}
$$

with $f_{z}(t)=\varepsilon M_{1} \int_{0}^{t} P_{z}(s) d s+\varepsilon\left(M_{2}-M_{1}\right) \int_{0}^{t} S_{z}(s) d s$. 
Let $G$ be given by

$$
\begin{aligned}
G: \mathcal{C}\left(I ; \mathbb{R}_{+}\right) & \longrightarrow \mathcal{C}\left(I ; \mathbb{R}_{+}\right) \\
z & \longmapsto G(z),
\end{aligned}
$$

where for each $t \in I$, we define

$$
G(z)(t)=\frac{Y_{0} e^{m t} \exp \left[f_{z}(t)\right]}{1+n Y_{0} \int_{0}^{t} e^{m \tau} \exp \left[f_{z}(\tau)\right] d \tau}
$$

It is clear that $G$ is well defined.

Then, we will prove that (9) has a unique solution and $G$ has a unique fixed point which gives existence and uniqueness of solution of the model (3).

\section{An age-structured epidemic model with different death-rates}

In this Section we study (9). We notice that this system is an age-structured epidemic model $s \rightarrow i \rightarrow s$ type where $\mu_{1, z}$ and $\mu_{2, z}$ are the age-specific mortality rate by the infective and susceptible individuals, respectively. In our knowledge, only models with the same rate of mortality of susceptible and infective individuals have been studied (for instance $[9,6,7]$ ). In our case, they are different. This leads us to a coupled system of equations and we do not have an equation depending only on a single variable.

Therefore, in this Section, we will study a model as (2), being $\hat{\mu}_{i}, i=1,2$, the death rates for infective and susceptible, respectively. We assume that $\hat{\mu}_{1} \geq \hat{\mu}_{2}$, and (H1) holds for $\hat{\mu}_{i}$. The existence and the uniqueness for (9) will follow from the similar properties for $(2)$.

In order to facilitate some useful estimates, we perform in (2) the change $i(a, t)=p(a, t)-s(a, t)$. Then (2) becomes

$$
\left\{\begin{array}{l}
\frac{\partial p}{\partial t}+\frac{\partial p}{\partial a}+\hat{\mu}_{1}(a, t, P(t)) p+\left(\hat{\mu}_{2}(a, t, P(t))-\hat{\mu}_{1}(a, t, P(t)) s=0\right. \\
\frac{\partial s}{\partial t}+\frac{\partial s}{\partial a}+\left(\hat{\mu}_{2}(a, t, P(t))+\gamma(a)\right) s=\lambda(a, t ; s-p) s+\gamma(a) p(a, t) \\
p(a, 0)=p_{0}(a), \quad s(a, 0)=s_{0}(a) \\
p(0, t)=\int_{0}^{\infty} \beta(a, t, P(t)) p(a, t) d a \\
s(0, t)=\int_{0}^{\infty} \beta(a, t, P(t))(q s(a, t)+(1-q) p(a, t)) d a \\
p(a, t), s(a, t) \longrightarrow 0, \text { when } a \rightarrow \infty
\end{array}\right.
$$

where $\rho_{0}(a):=\left(p_{0}(a), s_{0}(a)\right)=\left(i_{0}(a)+s_{0}(a), s_{0}(a)\right)$ a.e. $a \in(0, \infty)$. 
For biological reasons we are interested in nonnegative solutions, so that we consider that $p(a, t) \geq s(a, t)$. So, we will look for solutions of (13) belonging to the space

$$
V:=\left\{\rho \in L^{\infty}\left(I ;\left(L^{1}\left(\mathbb{R}_{+}\right)\right)^{2}\right) \mid \rho_{1}(a, t) \geq \rho_{2}(a, t) \geq 0 \text { a.e. }(a, t) \in \mathbb{R}_{+} \times I\right\} .
$$

On $V$, we take the norm

$$
|\rho|_{V}=\operatorname{ess} \sup _{t \in I} e^{-k t}|\rho(\cdot, t)|_{1}
$$

where $k$ is a positive constant which will be chosen later and $|\cdot|_{1}$ denotes the usual norm in $\left(L^{1}\left(\mathbb{R}_{+}\right)\right)^{2}$, i.e. $|\rho(\cdot, t)|_{1}=\left\|\rho_{1}(\cdot, t)\right\|_{L^{1}}+\left\|\rho_{2}(\cdot, t)\right\|_{L^{1}}$.

Namely, by a solution of $(13)$, we mean a function $\rho(\cdot, \cdot)=(p(\cdot, \cdot), s(\cdot, \cdot)) \in V$ such that

$$
\left\{\begin{array}{l}
D p=-\hat{\mu}_{1}(a, t, P(t)) p(a, t)-\left(\hat{\mu}_{2}(a, t, P(t))-\hat{\mu}_{1}(a, t, P(t)) s(a, t),\right. \\
D s=-\left(\hat{\mu}_{2}(a, t, P(t))+\gamma(a)-\lambda(a, t ; s-p)\right) s(a, t)+\gamma(a) p(a, t), \\
p(a, 0)=p_{0}(a), \quad s(a, 0)=s_{0}(a), \\
\lim _{h \rightarrow 0^{+}} p(0, t+h)=\int_{0}^{\infty} \beta(a, t, P(t)) p(a, t) d a, \\
\lim _{h \rightarrow 0^{+}} s(0, t+h)=\int_{0}^{\infty} \beta(a, t, P(t))(q s(a, t)+(1-q) p(a, t)) d a, \\
p(a, t), s(a, t) \longrightarrow 0, \text { when } a \rightarrow \infty,
\end{array}\right.
$$

where $D p$ and $D s$ denote the directional derivatives of $p$ and $s$ respectively, i.e.

$$
D p(a, t)=\lim _{h \rightarrow 0} \frac{p(a+h, t+h)-p(a, t)}{h} .
$$

Generally $\rho$ will not be differentiable everywhere; of course, when this occurs $D p=p_{a}+p_{t}, D s=s_{a}+s_{t}$.

Remark 1 Our solutions will be considered in the sense of (15). So that, it is not required that $\rho$ possesses partial derivatives with respect to a and $t$, but only the directional derivative. We must add conditions of regularity for the initial data and the compatibility conditions for $\rho_{0}$ that $\rho$ might be continuous in $a$ and be differentiable (see, for the case of an equation, [3]).

\subsection{Analysis of system (15)}

If we assume that $\rho:=(p, s)$ is smooth along the characteristics $a=t+k$ (except perhaps for a zero-measure set of $k$ (see [3])), then adding in both sides of $(15)_{1} \gamma(a)$ and integrating this equality and $(15)_{2}$ along these lines, 
we obtain an o.d.s. If we denote

$$
\begin{aligned}
& B_{u}(t)=\int_{0}^{\infty} \beta(\sigma, t, U(t)) u(\sigma, t) d \sigma \\
& B_{u, v}(t)=\int_{0}^{\infty} \beta(\sigma, t, U(t))(q v(\sigma, t)+(1-q) u(\sigma, t)) d \sigma,
\end{aligned}
$$

where $U(t)=\int_{0}^{\infty} u(a, t) d a$, then, solving this o.d.s., we have

$$
p(a, t)=\left\{\begin{array}{cc}
p_{0}(a-t) \pi(a, t, t ; \rho)+\int_{0}^{t} \pi(a, t, \sigma ; \rho)(\hat{\mu}(a-\sigma, t-\sigma, P(t-\sigma)) \\
\quad \times s(a-\sigma, t-\sigma)+\gamma(a-\sigma) p(a-\sigma, t-\sigma)) d \sigma \quad \text { if } a \geq t \\
B_{p}(t-a) \pi(a, t, a ; \rho)+\int_{0}^{a} \pi(a, t, \sigma ; \rho)(\hat{\mu}(a-\sigma, t-\sigma, P(t-\sigma)) \\
\quad \times s(a-\sigma, t-\sigma)+\gamma(a-\sigma) p(a-\sigma, t-\sigma)) d \sigma \quad \text { if } t>a
\end{array}\right.
$$

and

$s(a, t)=\left\{\begin{array}{l}s_{0}(a-t) \tilde{\pi}(a, t, t ; \rho)+\int_{0}^{t} \tilde{\pi}(a, t, \sigma ; \rho) \gamma(a-\sigma) p(a-\sigma, t-\sigma) d \sigma \text { if } a \geq t \\ B_{p, s}(t-a) \tilde{\pi}(a, t, a ; \rho)+\int_{0}^{a} \tilde{\pi}(a, t, \sigma ; \rho) \gamma(a-\sigma) p(a-\sigma, t-\sigma) d \sigma \text { if } t>a\end{array}\right.$

where: $\hat{\mu}(a, t, P(t))=\hat{\mu}_{1}(a, t, P(t))-\hat{\mu}_{2}(a, t, P(t))$,

$$
\begin{aligned}
& \pi(a, t, x ; \rho)=e^{\left(-\int_{0}^{x}\left(\hat{\mu}_{1}(a-\sigma, t-\sigma, P(t-\sigma))+\gamma(a-\sigma)\right) d \sigma\right)}, \\
& \tilde{\pi}(a, t, x ; \rho)=e^{\left(-\int_{0}^{x} \hat{\mu}_{2}(a-\sigma, t-\sigma, P(t-\sigma))+\lambda(a-\sigma, t-\sigma ; p-s)+\gamma(a-\sigma) d \sigma\right)} .
\end{aligned}
$$

We easily see that to solve (15) is equivalent to finding a solution of (16) and (17) (see [3]). So that, in the sequel, we restrict our attention to these integral equations.

Lemma 2 Suppose (H1)-(H5). For each $\rho_{0}=\left(p_{0}, s_{0}\right) \in\left(L^{1}\left(\mathbb{R}_{+}\right)\right)^{2}$ with $p_{0} \geq$ $s_{0}$, we denote $r=\log \left(\left|\rho_{0}\right|_{1}+e\right)$. If $\rho=(p, s) \in V$ satisfies (16) and (17), then there exists a constant $C$, depending only on $T$ and $\gamma_{\infty}$, such that

$$
|\rho(\cdot, t)|_{1} \leq \exp \left(r e^{C t}\right) \text { a.e. } t \in I .
$$

PROOF. Let $\rho=(p, s) \in V$ satisfy the above assumptions. Since $\rho \in V$ then $|\pi(a, t, x ; \rho)|,|\tilde{\pi}(a, t, x ; \rho)| \leq 1$. Hence, considering (16) and (17), and an obvious change of variables in the integrals, we have for almost all $t \in I$

$$
\begin{aligned}
& |\rho(\cdot, t)|_{1} \leq \mathrm{d} \int_{t}^{\infty}\left|\rho_{0}(a-t)\right| d a+2 \int_{0}^{t}\left[\int_{0}^{\infty}|\gamma(a)||p(a, \sigma)| d a\right] d \sigma \\
& \quad+\int_{0}^{t}\left[\int_{0}^{\infty}|\hat{\mu}(a, \sigma, P(\sigma))||s(a, \sigma)| d a\right] d \sigma+2 \int_{0}^{t}\left[\int_{0}^{\infty}|\beta(a, \sigma, P(\sigma))||\rho(a, \sigma)| d a\right] d \sigma .
\end{aligned}
$$


Since $\log (|P(u)|+e) \geq 1$, using (5) and (7), we get

$$
|\rho(\cdot, t)|_{1} \leq\left|\rho_{0}\right|_{1}+C \int_{0}^{t} \log (|P(u)|+e)|\rho(\cdot, u)|_{1} d u
$$

where $C \equiv C\left(T, \gamma_{\infty}\right)>0$.

We can now proceed analogously to the proof of [11, Lemma 1, pag. 19] and we obtain the result.

Let us $\rho=\left(\rho_{1}, \rho_{2}\right) \in V$, consider the map $\rho=\left(\rho_{1}, \rho_{2}\right) \in V \mapsto F(\rho)=$ $\left(F_{1}(\rho), F_{2}(\rho)\right) \in V$ where $F(\rho)$ is defined by

$F_{1}(\rho)(a, t)=\left\{\begin{array}{c}p_{0}(a-t) \pi(a, t, t ; \rho)+\int_{0}^{t} \pi(a, t, \sigma ; \rho)(\hat{\mu}(a-\sigma, t-\sigma, P(t-\sigma)) \\ \left.\quad \times F_{2}(\rho)(a-\sigma, t-\sigma)+\gamma(a-\sigma) \rho_{1}(a-\sigma, t-\sigma)\right) d \sigma \text { if } a \geq t \\ B_{\rho_{1}}(t-a) \pi(a, t, a ; \rho)+\int_{0}^{a} \pi(a, t, \sigma ; \rho)(\hat{\mu}(a-\sigma, t-\sigma, P(t-\sigma)) \\ \left.\times F_{2}(\rho)(a-\sigma, t-\sigma)+\gamma(a-\sigma) \rho_{1}(a-\sigma, t-\sigma)\right) d \sigma \text { if } t>a\end{array}\right.$

and

$$
F_{2}(\rho)(a, t)=\left\{\begin{aligned}
s_{0}(a-t) \tilde{\pi}(a, t, t ; \rho) \\
\quad+\int_{0}^{t} \tilde{\pi}(a, t, \sigma ; \rho) \gamma(a-\sigma) \rho_{1}(a-\sigma, t-\sigma) d \sigma \quad \text { if } a \geq t \\
B_{\rho_{1}, \rho_{2}}(t-a) \tilde{\pi}(a, t, a ; \rho) \\
\quad+\int_{0}^{a} \tilde{\pi}(a, t, \sigma ; \rho) \gamma(a-\sigma) \rho_{1}(a-\sigma, t-\sigma) d \sigma \text { if } t>a
\end{aligned}\right.
$$

where $P(t):=\int_{0}^{\infty} \rho_{1}(a, t) d a$.

Lemma 3 Under the assumption of Lemma 2, we have $F: V \longrightarrow V$.

\section{PROOF.}

Remark 4 Throughout this proof, for abbreviation, we write

$$
\Sigma(x, y):=e^{-\int_{x}^{y} \hat{\mu}(a-\tau, t-\tau, P(t-\tau)) d \tau}, \Psi(x, y):=e^{-\int_{x}^{y} \lambda\left(a-\tau, t-\tau ; \rho_{1}-\rho_{2}\right) d \tau} .
$$

If $\rho \in V$, then $P(t) \in L^{\infty}(I)$. Thus by (4) and (6) we have that $\beta(a, t, P(t))$, $\hat{\mu}(a, t, P(t)) \in L^{\infty}\left(\mathbb{R}_{+} \times I\right)$. Hence, $F$ is clearly measurable in $a$ and essentially bounded on $I$.

By (22), we have $F_{2}(\rho)(a, t) \geq 0$ a.e. $(a, t) \in \mathbb{R}_{+} \times I$. So, we only need to show that $F_{1}(\rho)(a, t) \geq F_{2}(\rho)(a, t)$ a.e. $(a, t) \in \mathbb{R}_{+} \times I$. 
We assume that $a \geq t$ (the discussion for $a<t$ is similar), using (21) and (22), and substituting $F_{2}$ into $F_{1}$ we get,

$$
\begin{aligned}
& F_{1}(\rho)(a, t)-F_{2}(\rho)(a, t)=p_{0}(a-t) \pi(a, t, t ; \rho)-s_{0}(a-t) \tilde{\pi}(a, t, t ; \rho) \\
& +\int_{0}^{t} \pi(a, t, \sigma ; \rho) \hat{\mu}(a-\sigma, t-\sigma, P(t-\sigma)) s_{0}(a-t) \tilde{\pi}(a-\sigma, t-\sigma, t-\sigma ; \rho) d \sigma \\
& +\int_{0}^{t} \pi(a, t, \sigma ; \rho) \hat{\mu}(a-\sigma, t-\sigma, P(t-\sigma)) \\
& \quad \times\left(\int_{\sigma}^{t} \tilde{\pi}(a-\sigma, t-\sigma, \tau-\sigma ; \rho) \gamma(a-\tau) \rho_{1}(a-\tau, t-\tau) d \tau\right) d \sigma \\
& +\int_{0}^{t} \pi(a, t, \sigma ; \rho) \gamma(a-\sigma) \rho_{1}(a-\sigma, t-\sigma) d \sigma \\
& -\int_{0}^{t} \tilde{\pi}(a, t, \sigma ; \rho) \gamma(a-\sigma) \rho_{1}(a-\sigma, t-\sigma) d \sigma:=A-B+C+D+E-F .
\end{aligned}
$$

- Estimation $A-B+C$ : By the mean value theorem, there exists $t_{1} \in(0, t)$, such that

$$
C=s_{0}(a-t) \tilde{\pi}(a, t, t ; \rho)(1-\Sigma(0, t))\left(\Psi\left(0, t_{1}\right)\right)^{-1} .
$$

So,

$$
\begin{aligned}
A-B+C & \geq p_{0}(a-t) \pi(a, t, t ; \rho)-s_{0}(a-t) \pi(a, t, t ; \rho) \Psi\left(t_{1}, t\right) \\
& \geq s_{0}(a-t) \pi(a, t, t ; \rho)\left(1-\Psi\left(t_{1}, t\right)\right) \geq 0 .
\end{aligned}
$$

- Estimation $D+E-F$ : Interchanging the order of integration in $\tau$ and $\sigma$, and applying the mean value theorem, with $t_{\tau} \in(0, \tau)$ we obtain

$$
\begin{aligned}
D & =\int_{0}^{t} \Sigma(0, \tau) e^{-\int_{0}^{\tau} \gamma(a-\delta) d \delta} \gamma(a-\tau) \rho_{1}(a-\tau, t-\tau)\left(\int_{0}^{\tau}-\frac{d}{d \sigma}(\Sigma(0, \sigma)) \Psi(\sigma, \tau) d \sigma\right) d \tau \\
& =\int_{0}^{t}\left((1-\Sigma(0, \tau)) \Psi\left(t_{\tau}, \tau\right) \Sigma(0, \tau) e^{-\int_{0}^{\tau} \gamma(a-\delta) d \delta} \gamma(a-\tau) \rho_{1}(a-\tau, t-\tau)\right) d \tau
\end{aligned}
$$

Hence,

$$
\begin{aligned}
D+E & -F=\int_{0}^{t} \tilde{\pi}(a, t, \tau ; \rho) \gamma(a-\tau) \rho_{1}(a-\tau, t-\tau)\left(\Psi\left(0, t_{\tau}\right)^{-1}-1\right) d \tau \\
& +\int_{0}^{t} \pi(a, t, \tau ; \rho) \gamma(a-\tau) \rho_{1}(a-\tau, t-\tau)\left(1-\Psi\left(t_{\tau}, \tau\right)\right) d \tau \geq 0
\end{aligned}
$$

So that $F_{1}(\rho)(a, t) \geq F_{2}(\rho)(a, t) \geq 0$ a.e. $a \in(t, \infty)$.

From which we can conclude that for each $\rho \in V, F(\rho) \in V$.

The following result provides us with some useful estimates. 
Lemma 5 Under the above assumptions, let $r$ be as the Lemma 2, i.e. $r=$ $\log \left(\left|\rho_{0}\right|_{1}+e\right)$ and $w>0$. Consider the set

$$
C_{r, \omega}=\mathrm{d}\left\{\left.\rho \in V|| \rho(\cdot, t)\right|_{1} \leq \exp \left(r e^{\omega t}\right) \text { a.e. } t \in I\right\} .
$$

Let $\rho:=(p, s), \rho^{\prime}:=\left(p^{\prime}, s^{\prime}\right) \in C_{r, \omega}, a \in \mathbb{R}_{+}, t \in I$. Then for $x \leq \min \{a, t\}$

$|\tilde{\pi}(a, t, x ; \rho)| \leq 1, \quad|\pi(a, t, x ; \rho)| \leq 1$.

$\exists M(T)>0$ such that $|P(t)|,\left|B_{p}(t)\right|,\left|B_{p, s}(t)\right| \leq M$ a.e. $t \in I$.

$\exists C(T)>0$ such that $\left|\pi(a, t, x ; \rho)-\pi\left(a, t, x ; \rho^{\prime}\right)\right| \leq \frac{C}{k}\left|\rho-\rho^{\prime}\right|_{V} e^{k t}$.

$\exists C\left(T, K_{\infty}\right)>0$ such that

$$
\begin{aligned}
& \left|\tilde{\pi}(a, t, x ; \rho)-\tilde{\pi}\left(a, t, x ; \rho^{\prime}\right)\right| \leq \frac{C}{k}\left|\rho-\rho^{\prime}\right|_{V} e^{k t} \\
& \quad+\theta_{\infty} \int_{0}^{x}\left|\rho(a-\sigma, t-\sigma)-\rho^{\prime}(a-\sigma, t-\sigma)\right| d \sigma .
\end{aligned}
$$

PROOF. Firstly, note that (27) and (28) are immediate. Let us to prove (29) and (30), using the inequality $\left|e^{-x}-e^{-y}\right| \leq|x-y| \forall x, y \in \mathbb{R}_{+}$, we have

$$
\begin{aligned}
& \left|\tilde{\pi}(a, t, x ; \rho)-\tilde{\pi}\left(a, t, x ; \rho^{\prime}\right)\right| \\
& \leq \int_{0}^{x}\left|\hat{\mu}_{2}(a-\sigma, t-\sigma, P(t-\sigma))-\hat{\mu}_{2}\left(a-\sigma, t-\sigma, P^{\prime}(t-\sigma)\right)\right| d \sigma \\
& \quad+\int_{0}^{x}\left|\lambda(a-\sigma, t-\sigma ; p-s)-\lambda\left(a-\sigma, t-\sigma ; p^{\prime}-s^{\prime}\right)\right| d \sigma \\
& \leq C \int_{t-x}^{t}\left|\rho(\cdot, \sigma)-\rho^{\prime}(\cdot, \sigma)\right|_{1} d \sigma+\theta_{\infty} \int_{0}^{x}\left|\rho(a-\sigma, t-\sigma)-\rho^{\prime}(a-\sigma, t-\sigma)\right| d \sigma \\
& \leq \frac{C}{k}\left|\rho-\rho^{\prime}\right|_{V} e^{k t}+\theta_{\infty} \int_{0}^{x}\left|\rho(a-\sigma, t-\sigma)-\rho^{\prime}(a-\sigma, t-\sigma)\right| d \sigma .
\end{aligned}
$$

An analogous estimate to above one implies (29).

Theorem 6 Under the above assumptions, for each $T>0$ and for each $\rho_{0}=$ $\left(p_{0}, s_{0}\right) \in\left(L^{1}\left(\mathbb{R}_{+}\right)\right)^{2}$, with $p_{0} \geq s_{0}$, there exists a unique $\rho=(p, s) \in V$ satisfying (16) and (17). And so, we have that $\rho$ is the unique solution of problem (13).

PROOF. To prove the result, it remains to show that $F$ (defined by (21) and (22)) has a unique point fixed in $V$.

Let $C_{r, \omega}$ define by (26), then for $\omega$ great enough $F$ maps $C_{r, \omega}$ into $C_{r, \omega}$. Indeed, by (20), we get, for almost all $t \in I$

$$
|F(\rho)(\cdot, t)|_{1} \leq\left|\rho_{0}\right|_{1}+C \int_{0}^{t} \log \left(|\rho(\cdot, u)|_{1}+e\right)|\rho(\cdot, u)|_{1} d u
$$


and from [11, proof Th. 2, pag. 20] it follows that

$$
|F(\rho)(\cdot, t)|_{1} \leq \exp \left(r e^{\omega t}\right) \text { a.e. } t \in I
$$

for $\omega>0$ depending on $T$ and on $\gamma_{\infty}$. Hence, we have proved that $F$ maps $C_{r, \omega}$ into $C_{r, \omega}$.

Let us assume $\omega$ fixed such that $F(\rho)$ remains in $C_{r, \omega}$ for $\rho$ in $C_{r, \omega}$. Clearly, $C_{r, \omega}$ is closed in $V$ and to prove that $F$ has a unique fixed point in $C_{r, \omega}$, it suffices to prove that $F$ is a strict contraction, for instance for the norm defined in (14) with $k$ suitable.

For $\rho:=(p, s), \rho^{\prime}:=\left(p^{\prime}, s^{\prime}\right) \in C_{r, \omega}$, let us estimate $\left|F(\rho)-F\left(\rho^{\prime}\right)\right|_{V}$.

First, for almost all $t \in I$,

$$
\begin{aligned}
\left|F(\rho)(\cdot, t)-F\left(\rho^{\prime}\right)(\cdot, t)\right|_{1}= & \int_{0}^{\infty}\left|F_{1}(\rho)(a, t)-F_{1}\left(\rho^{\prime}\right)(a, t)\right| d a \\
& +\int_{0}^{\infty}\left|F_{2}(\rho)(a, t)-F_{2}\left(\rho^{\prime}\right)(a, t)\right| d a:=\widetilde{I}_{1}+\widetilde{I}_{2} .
\end{aligned}
$$

Now, substituting the expression of $F_{1}$ into $\widetilde{I}_{1}$, we get,

$$
\begin{aligned}
& \widetilde{I}_{1} \leq \int_{0}^{t}\left|B_{p}(t-a) \pi(a, t, a ; \rho)-B_{p^{\prime}}(t-a) \pi\left(a, t, a ; \rho^{\prime}\right)\right| d a \\
& +\int_{t}^{\infty}\left|p_{0}(a-t)\right|\left|\pi(a, t, t ; \rho)-\pi\left(a, t, t ; \rho^{\prime}\right)\right| d a \\
& +\int_{0}^{t}\left(\int_{0}^{\infty} \mid \pi(a+t-\sigma, t, t-\sigma ; \rho) \hat{\mu}(a, \sigma, P(\sigma)) F_{2}(\rho)(a, \sigma)\right. \\
& \left.\quad-\pi\left(a+t-\sigma, t, t-\sigma ; \rho^{\prime}\right) \hat{\mu}\left(a, \sigma, P^{\prime}(\sigma)\right) F_{2}\left(\rho^{\prime}\right)(a, \sigma) \mid d a\right) d \sigma \\
& +\gamma_{\infty} \int_{0}^{t}\left(\int_{0}^{\infty}\left|\pi(a+t-\sigma, t, t-\sigma ; \rho) p(a, \sigma)-\pi\left(a+t-\sigma, t, t-\sigma ; \rho^{\prime}\right) p^{\prime}(a, \sigma)\right| d a\right) d \sigma \\
& \quad:=I_{1}+I_{2}+I_{3}+I_{4},
\end{aligned}
$$

where $P$ and $P^{\prime}$ is defined by $\int_{0}^{\infty} p(a, t) d a$ and $\int_{0}^{\infty} p^{\prime}(a, t) d a$, respectively.

Hence $\left|F(\rho)(\cdot, t)-F\left(\rho^{\prime}\right)(\cdot, t)\right|_{1} \leq I_{1}+I_{2}+I_{3}+I_{4}+\widetilde{I}_{2}$.

- Estimate of $I_{2} \cdot-$ By $(29)$, we have

$$
I_{2} \leq \frac{C}{k}\left|\rho-\rho^{\prime}\right|_{V} e^{k t} \int_{t}^{\infty}\left|p_{0}(a-t)\right| d a \leq \frac{C\left|\rho_{0}\right|_{1}}{k}\left|\rho-\rho^{\prime}\right|_{V} e^{k t} .
$$

- Estimate of $I_{1}$ -

$$
\begin{aligned}
I_{1} \leq \int_{0}^{t} & \left|B_{p}(t-a)\right|\left|\pi(a, t, a ; \rho)-\pi\left(a, t, a ; \rho^{\prime}\right)\right| d a \\
& +\int_{0}^{t}\left|B_{p}(t-a)-B_{p^{\prime}}(t-a)\right|\left|\pi\left(a, t, a ; \rho^{\prime}\right)\right| d a:=I_{1}^{1}+I_{1}^{2} .
\end{aligned}
$$


Let us now estimate $I_{1}^{1}$, by (28) and (29), we get

$$
I_{1}^{1} \leq \frac{M C(T)}{k}\left|\rho-\rho^{\prime}\right|_{V} e^{k t} .
$$

Let us estimate $I_{1}^{2}$; by $(27)$

$$
\begin{aligned}
I_{1}^{2} \leq & \int_{0}^{t}\left(\int_{0}^{\infty}|\beta(\xi, u, P(u))|\left|p(\xi, u)-p^{\prime}(\xi, u)\right| d \xi\right) d u \\
& +\int_{0}^{t}\left(\int_{0}^{\infty}\left|\beta(\xi, u, P(u))-\beta\left(\xi, u, P^{\prime}(u)\right)\right|\left|p^{\prime}(\xi, u)\right| d \xi\right) d u \leq \frac{M}{k}\left|\rho-\rho^{\prime}\right|_{V} e^{k t},
\end{aligned}
$$

using (7) and (28) in the first term and (6) and the fact that $\rho^{\prime} \in C_{r, \omega}$ in the second one.

- Estimate of $\widetilde{I}_{2}$-- Substituting the expression for $F_{2}$ into $\widetilde{I}_{2}$, and applying (30) in the first term, (30) in the second one and proceeding analogously to the third one, we obtain

$$
\begin{aligned}
\widetilde{I}_{2} & \leq \int_{0}^{t}\left|B_{p, s}(t-a) \tilde{\pi}(a, t, a ; \rho)-B_{p^{\prime}, s^{\prime}}(t-a) \tilde{\pi}\left(a, t, a ; \rho^{\prime}\right)\right| d a \\
& +\int_{0}^{t} \int_{0}^{\infty}\left|\tilde{\pi}(a+t-\sigma, t, t-\sigma ; \rho) p(a, \sigma)-\tilde{\pi}\left(a+t-\sigma, t, t-\sigma ; \rho^{\prime}\right) p^{\prime}(a, \sigma)\right| d a d \sigma \\
& +\int_{t}^{\infty}\left|s_{0}(a-t)\right|\left|\tilde{\pi}(a, t, t ; \rho)-\tilde{\pi}\left(a, t, t ; \rho^{\prime}\right)\right| d a \leq \frac{M}{k}\left|\rho-\rho^{\prime}\right|_{V} e^{k t}
\end{aligned}
$$

- Estimate of $I_{3 \cdot-}$ By (5) and (28) we have that $|\hat{\mu}(a, t, P(t))| \leq \bar{\mu}$, then

$$
\begin{aligned}
I_{3} & \leq \bar{\mu} \int_{0}^{t} \int_{0}^{\infty}|\pi(a+t-\sigma, t, t-\sigma ; \rho)|\left|F_{2}(\rho)(a, \sigma)-F_{2}\left(\rho^{\prime}\right)(a, \sigma)\right| d a d \sigma \\
& +\bar{\mu} \int_{0}^{t} \int_{0}^{\infty}\left|\pi(a+t-\sigma, t, t-\sigma ; \rho)-\pi\left(a+t-\sigma, t, t-\sigma ; \rho^{\prime}\right)\right|\left|F_{2}\left(\rho^{\prime}\right)(a, \sigma)\right| d a d \sigma \\
& +\int_{0}^{t} \int_{0}^{\infty}\left|\pi\left(a+t-\sigma, t, t-\sigma ; \rho^{\prime}\right)\right|\left|F_{2}\left(\rho^{\prime}\right)(a, \sigma)\right|\left|\hat{\mu}(a, \sigma, P(\sigma))-\hat{\mu}\left(a, \sigma, P^{\prime}(\sigma)\right)\right| d a d \sigma
\end{aligned}
$$

But $\left\|F_{2}(\rho)(\cdot, \sigma)\right\|_{L^{1}} \leq M$ since $F(\rho) \in C_{r, \omega}$. Then, estimations similar to the above imply that

$$
I_{3} \leq\left(\frac{M}{k^{2}}+\frac{M}{k}\right)\left|\rho-\rho^{\prime}\right|_{V} e^{k t} .
$$

- Estimate of $I_{4} \cdot-$ In the same manner we can see that

$$
I_{4} \leq \frac{M}{k}\left|\rho-\rho^{\prime}\right|_{V} e^{k t}
$$

Therefore, joining all estimations, we get that for almost all $t \in I$, there exists two constants $M$ and $\tilde{M}$ depending only on $\rho_{0}, T$ and on $\gamma_{\infty}, \theta_{\infty}$ and $K_{\infty}$, such that

$$
\left|F(\rho)(\cdot, t)-F\left(\rho^{\prime}\right)(\cdot, t)\right|_{1} \leq\left(\frac{M}{k}+\frac{\tilde{M}}{k^{2}}\right)\left|\rho-\rho^{\prime}\right|_{V} e^{k t}
$$


Dividing both sides of this inequality by $e^{k t}$, we obtain

$$
\left|F(\rho)-F\left(\rho^{\prime}\right)\right|_{V} \leq\left(\frac{M}{k}+\frac{\tilde{M}}{k^{2}}\right)\left|\rho-\rho^{\prime}\right|_{V} .
$$

And thus for $k$ great enough $F$ is a strict contraction with a unique fixed point in $C_{r, \omega}$, and so in $V$. This concludes the proof.

\section{Existence and uniqueness of solution of the model (3)}

Under the assumptions of Section 1 , it is easily seen that for each $z \in \mathcal{C}\left(I ; \mathbb{R}_{+}\right)$, $\mu_{i, z}(i=1,2)$ (defined in (8)) satisfy the hypotheses of existence and uniqueness of a solution of the problem (9). So that, for each $z \in \mathcal{C}\left(I ; \mathbb{R}_{+}\right)$, we have that there exists a unique nonnegative solution $\left(i_{z}, s_{z}\right) \in L^{\infty}\left(I ;\left(L^{1}\left(\mathbb{R}_{+}\right)\right)^{2}\right)$.

Hence, to finish the study of model (3), we only need to prove that the map $G$ defined by (12) has a unique fixed point in $\mathcal{C}\left(I ; \mathbb{R}_{+}\right)$. For that, we consider $\mathcal{C}\left(I ; \mathbb{R}_{+}\right)$endowed with Bielecki's norm, which it will be denoted by $\|\cdot\|_{B}$.

Lemma 7 For each $z \in \mathcal{C}\left(I ; \mathbb{R}_{+}\right)$, we have that $P_{z}$ is essentially bounded by a constant independent of $z$.

Remark 8 Lemma 7 is not a consequence of Lemma 2, since if we apply the proof of the Lemma 2 to $\mu_{i, z}$, we obtain a bound depending on the supreme of $z$.

PROOF. Let $t \in I$, and $z \in \mathcal{C}\left(I ; \mathbb{R}_{+}\right)$. Consider $\rho_{z}:=\left(p_{z}, s_{z}\right)$ the unique solution of problem (9). We define

$$
\begin{aligned}
& \tilde{\pi}\left(a, t, x ; \rho_{z}\right):=e^{-\int_{0}^{x}\left(\mu_{2, z}\left(a-\sigma, t-\sigma, P_{z}(t-\sigma)\right) \lambda\left(a-\sigma, t-\sigma ; p_{z}-s_{z}\right)+\gamma(a-\sigma)\right) d \sigma}, \\
& \pi\left(a, t, x ; \rho_{z}\right):=e^{-\int_{0}^{x}\left(\mu_{1, z}\left(a-\sigma, t-\sigma, P_{z}(t-\sigma)\right)+\gamma(a-\sigma)\right) d \sigma} \\
& \mu_{z}\left(a, t, P_{z}(t)\right):=\mu_{1, z}\left(a, t, P_{z}(t)\right)-\mu_{2, z}\left(a, t, P_{z}(t)\right) .
\end{aligned}
$$

Observe that $\left(p_{z}, s_{z}\right)$ verifies (16) and (17), with $\pi$ and $\tilde{\pi}$ defined in (38) and (37), respectively, and changing $\hat{\mu}$ by $\mu_{z}$ defined in (39). Consequently,

$$
\begin{gathered}
\left|P_{z}(t)\right| \leq \int_{0}^{t}\left|B_{p_{z}}(t-a)\right| d a+\left\|p_{0}\right\|_{L^{1}}+\gamma_{\infty} \int_{0}^{t}\left(\int_{0}^{\infty}\left|p_{z}(a, \sigma)\right| d a\right) d \sigma \\
+\int_{0}^{t}\left(\int_{0}^{a}\left|\pi\left(a, t, \sigma ; \rho_{z}\right)\right|\left|\mu_{z}\left(a-\sigma, t-\sigma, P_{z}(t-\sigma)\right)\right|\left|s_{z}(a-\sigma, t-\sigma)\right| d \sigma\right) d a \\
+\int_{t}^{\infty}\left(\int_{0}^{t}\left|\pi\left(a, t, \sigma ; \rho_{z}\right)\right|\left|\mu_{z}\left(a-\sigma, t-\sigma, P_{z}(t-\sigma)\right)\right|\left|s_{z}(a-\sigma, t-\sigma)\right| d \sigma\right) d a \\
:=K_{1}+K_{2}+K_{3}+K_{4}+K_{5} .
\end{gathered}
$$


Now, substituting the expression for $s_{z}$ into $K_{4}$ and $K_{5}$, we get

$$
K_{4}+K_{5} \leq L_{1}+L_{2}+L_{3}+L_{4},
$$

where

$$
\begin{aligned}
& L_{1}:=\int_{0}^{t} \int_{0}^{a} \pi\left(a, t, \sigma ; \rho_{z}\right) \mu_{z}\left(a-\sigma, t-\sigma, P_{z}(t-\sigma)\right) \\
& \times B_{p_{z}, s_{z}}(t-a) \tilde{\pi}\left(a-\sigma, t-\sigma, a-\sigma ; \rho_{z}\right) d \sigma d a, \\
& L_{2}:=\int_{0}^{t}\left(\int_{0}^{a} \pi\left(a, t, \sigma ; \rho_{z}\right)\left|\mu_{z}\left(a-\sigma, t-\sigma, P_{z}(t-\sigma)\right)\right|\right. \\
& \left.\times\left(\int_{\sigma}^{t} \tilde{\pi}\left(a-\sigma, t-\sigma, \eta-\sigma ; \rho_{z}\right)|\gamma(a-\eta)|\left|p_{z}(a-\eta, t-\eta)\right| d \eta\right) d \sigma\right) d a, \\
& L_{3}:=\int_{t}^{\infty} \int_{0}^{t} \pi\left(a, t, \sigma ; \rho_{z}\right) \mu_{z}\left(a-\sigma, t-\sigma, P_{z}(t-\sigma)\right) \\
& \times s_{0}(a-t) \tilde{\pi}\left(a-\sigma, t-\sigma, t-\sigma ; \rho_{z}\right) d \sigma d a, \\
& L_{4}:=\int_{t}^{\infty}\left(\int_{0}^{t} \pi\left(a, t, \sigma ; \rho_{z}\right)\left|\mu_{z}\left(a-\sigma, t-\sigma, P_{z}(t-\sigma)\right)\right|\right. \\
& \left.\times\left(\int_{\sigma}^{t} \tilde{\pi}\left(a-\sigma, t-\sigma, \eta-\sigma ; \rho_{z}\right)|\gamma(a-\eta)|\left|p_{z}(a-\eta, t-\eta)\right| d \eta\right) d \sigma\right) d a .
\end{aligned}
$$

So that, $\left|P_{z}(t)\right| \leq K_{1}+K_{2}+K_{3}+L_{1}+L_{2}+L_{3}+L_{4}$.

- The estimate $L_{3}$ is similar to $C$ in (23). Then by (24) and (27), we obtain

$$
L_{3} \leq\left\|s_{0}\right\|_{L^{1}} .
$$

- Estimate of $L_{1}$-- An analogous estimate to above one implies that

$$
L_{1} \leq \int_{0}^{t}\left|B_{s_{z}}(t-a)\right| d a .
$$

- $L_{2}$ and $L_{4}$ are similar to $D$ in (23). So that, by (25), we get

$$
L_{2}+L_{4} \leq \int_{0}^{t}\left(\int_{0}^{\infty}|\gamma(a)|\left|p_{z}(a, \sigma)\right| d a\right) d \sigma .
$$

Since, $s_{z} \leq p_{z}$, by (40)-(42), we get

$$
\left|P_{z}(t)\right| \leq\left|\rho_{0}\right|_{1}+2 \int_{0}^{t}\left|B_{p_{z}}(t-a)\right| d a+2 \gamma_{\infty} \int_{0}^{t}\left\|p_{z}(\cdot, \sigma)\right\|_{L^{1}} d \sigma .
$$

We can now proceed analogously to the proof of Lemma 2. Then, there exists a constant $C$ depending only on $T$ and on $\gamma_{\infty}$, such that if we denote by $r=\log \left(\left|\rho_{0}\right|_{1}+e\right)$, we obtain

$$
\left|P_{z}(t)\right| \leq \exp \left(r e^{C t}\right) \text { a.e. } t \in I \quad \forall z \in \mathcal{C}\left(I ; \mathbb{R}_{+}\right) .
$$


Remark 9 Since $S_{z}(t) \leq P_{z}(t)$ a.e. $t \in I$, then $S_{z}$ is also essentially bounded by a constant independent of $z$.

Lemma 10 Given $z, z^{\prime} \in \mathcal{C}\left(I ; \mathbb{R}_{+}\right)$, under the above notations, then there exists a constant $M<1$ such that

$$
\left|\rho_{z}-\rho_{z^{\prime}}\right|_{V} \leq M\left\|z-z^{\prime}\right\|_{B}
$$

PROOF. For almost all $t \in I,\left|\rho_{z}(\cdot, t)-\rho_{z^{\prime}}(\cdot, t)\right|_{1} \leq I_{1}+I_{2}+I_{3}+I_{4}+A^{*}$, where, $I_{1}, I_{2}, I_{3}$ and $I_{4}$ are defined in (31) and

$$
\begin{aligned}
A^{*}=\int_{0}^{t}\left(\int_{0}^{\infty} \mid\right. & \pi\left(a-t+\sigma, t, t-\sigma ; \rho_{z}\right) \mu_{z}\left(a, \sigma, P_{z}(\sigma)\right) s_{z}(a, \sigma) \\
& \left.-\pi\left(a-t+\sigma, t, t-\sigma ; \rho_{z^{\prime}}\right) \mu_{z^{\prime}}\left(a, \sigma, P_{z^{\prime}}(\sigma)\right) s_{z^{\prime}}(a, \sigma) \mid d a\right) d \sigma .
\end{aligned}
$$

In order to estimate $I_{2}$, for instance, we proceed analogously to (29). And we get

$$
\left|\pi\left(a, t, t ; \rho_{z}\right)-\pi\left(a, t, t ; \rho_{z^{\prime}}\right)\right| \leq \frac{C(T)}{k}\left|\rho_{z}-\rho_{z^{\prime}}\right|_{V} e^{k t}+\frac{M_{1}}{k}\left\|z-z^{\prime}\right\|_{B} e^{k t} .
$$

Hence, likewise to (32), we have

$$
I_{2} \leq \frac{C(T)\left|\rho_{0}\right|_{1}}{k}\left|\rho_{z}-\rho_{z^{\prime}}\right|_{V} e^{k t}+\frac{M_{1}\left|\rho_{0}\right|_{1}}{k}\left\|z-z^{\prime}\right\|_{B} e^{k t}
$$

Similar estimates to the proof of Lemma 7 imply that

$$
\left|\rho_{z}-\rho_{z^{\prime}}\right|_{V} \leq M\left\|z-z^{\prime}\right\|_{B}
$$

with $M<1$ for $k$ great enough.

Lemma 11 Under the above notations, for $k$ large enough, there exists a constant $M<1$ such that

$$
\underset{t \in I}{\operatorname{ess} \sup } e^{-k t}\left(\left|S_{z}(t)-S_{z^{\prime}}(t)\right|+\left|P_{z}(t)-P_{z^{\prime}}(t)\right|\right) \leq M\left\|z-z^{\prime}\right\|_{B}
$$

PROOF. The proof is straightforward from Lemma 10.

Theorem 12 Under the same hypotheses, G (defined in (12)) has a unique fixed point. So, we get existence and uniqueness of a solution of the problem (3). 
PROOF. Let us $t \in I$, for all $z \in \mathcal{C}\left(I ; \mathbb{R}_{+}\right)$throughout the proof we denote

$$
\Theta_{z}:=n Y_{0} \int_{0}^{t} e^{m \tau} \exp \left[f_{z}(\tau)\right] d \tau
$$

Consider $z_{1}, z_{2} \in \mathcal{C}\left(I ; \mathbb{R}_{+}\right)$, then

$$
\begin{aligned}
& \left|G\left(z_{1}\right)(t)-G\left(z_{2}\right)(t)\right|=\left|Y_{0} e^{m t}\left(\frac{\exp \left[f_{z_{1}}(t)\right]}{1+\Theta_{z_{1}}}-\frac{\exp \left[f_{z_{2}}(t)\right]}{1+\Theta_{z_{2}}}\right)\right| \\
& \left.\quad \leq\left|Y_{0}\right| e^{m t}\left(\left|e^{f_{z_{1}}(t)}-e^{f_{z_{2}}(t)}\right|+\mid e^{f_{z_{1}}(t)} \Theta_{z_{2}}-e^{f_{z_{2}}(t)} \Theta_{z_{1}}\right] \mid\right):=\left|Y_{0}\right| e^{m t}(A+B) .
\end{aligned}
$$

On the other hand, since $\left|e^{x}-1\right| \leq|x| e^{|x|}$ and by (43), we have that $f_{z_{i}}$ is bounded by a constant independent of $z_{i}$, then

$$
A=\exp \left[f_{z_{1}}(t)\right]\left|1-\exp \left[f_{z_{2}}(t)-f_{z_{1}}(t)\right]\right| \leq M\left|f_{z_{2}}(t)-f_{z_{1}}(t)\right| .
$$

Using (46), we get

$$
A \leq M \int_{0}^{t}\left(\left|S_{z_{1}}(s)-S_{z_{2}}(s)\right|+\left|P_{z_{1}}(s)-P_{z_{2}}(s)\right|\right) d s \leq \frac{M}{k}\left\|z_{1}-z_{2}\right\|_{B} e^{k t} .
$$

Let us now estimate $B$,

$$
B \leq n Y_{0} e^{f_{z_{1}}(t)} e^{f_{z_{2}}(t)} e^{m T}\left|\int_{0}^{t}\left(e^{f_{z_{2}}(\eta)} e^{-f_{z_{2}}(t)}-e^{f_{z_{1}}(\eta)} e^{-f_{z_{1}}(t)}\right) d \eta\right| .
$$

But,

$$
\exp \left[f_{z}(\eta)\right] \exp \left[-f_{z}(t)\right]=\exp \left[-\varepsilon\left(\int_{\eta}^{t} M_{1} P_{z}(s)+\left(M_{2}-M_{1}\right) S_{z}(s) d s\right)\right] .
$$

Since $\left|e^{-x}-e^{-y}\right| \leq|x-y|, \forall x, y \in \mathbb{R}_{+}$, we get

$$
\begin{aligned}
B \leq n Y_{0} e^{f_{z_{1}}(t)} e^{f_{z_{2}}(t)} e^{m T} \mid \varepsilon \int_{0}^{t} & \left(\int_{\eta}^{t} M_{1}\left|P_{z_{2}}(s)-P_{z_{1}}(s)\right|\right. \\
& \left.+\left(M_{2}-M_{1}\right)\left|S_{z_{2}}(s)-S_{z_{1}}(s)\right| d s\right) d \tau \mid
\end{aligned}
$$

And as in the estimate for $A$, we obtain

$$
B \leq \frac{C}{k}\left\|z_{1}-z_{2}\right\|_{B} e^{k t}
$$

So that,

hence

$$
\left|G\left(z_{1}\right)(t)-G\left(z_{2}\right)(t)\right| \leq \frac{M}{k}\left\|z_{1}-z_{2}\right\|_{B} e^{k t}
$$

$$
\left\|G\left(z_{1}\right)-G\left(z_{2}\right)\right\|_{B} \leq \frac{M}{k}\left\|z_{1}-z_{2}\right\|_{B}
$$

And thus, for $k$ great enough $G$ is a strict contraction. So, $G$ has a unique fixed point in $\mathcal{C}\left(I ; \mathbb{R}_{+}\right)$. This completes the proof. 


\section{References}

[1] A. J. Lotka, Elements of Mathematical Biology, Dover, 1956.

[2] H. Von Foerster, Some remarks on changing populations, The Kinetics of Cellular Proliferation (1959) 382-407.

[3] M. E. Gurtin, R. C. MacCamy, Non-linear age-dependent population dynamics, Arch. Ration. Mech. Anal. 54 (1974) 281-300.

[4] G. Webb, Theory of nonlinear Age-dependent Population Dynamics, Pure Appl. Math. Monographs, Marcel Dekker, New York, 1985.

[5] S. Busenberg, K. Cooke, M. Iannelli, Endemic threshold and stability in a class of age-structured epidemics, SIAM J. Appl. Math. 48 (1988) 1379-1395.

[6] M. E. Doma, Analysis of nonlinear integro-differential equations arising in agedependent epidemic models, Nonlinear Anal. 11 (1987) 913-937.

[7] M. Iannelli, M. Y. Kim, E. J. Park, Asymptotic behavior for an SIS epidemic model and its approximation, Nonlinear Anal. 35 (1999) 797-814.

[8] J. Chattopadhyay, O. Arino, A predator-prey model with disease in the prey, Nonlinear Anal. 36 (1999) 747-766.

[9] S. Busenberg, M. Iannelli, H. R. Thieme, Global behavior of an age-structured epidemic model, SIAM J. Math. Anal. 22 (1991) 1065-1080.

[10] P. Magal, Compact attractors for time-periodic age-structured population models, Elect. J. Diff. Eqn. 65 (2001) 1-35.

[11] M. Chipot, On the equations of age-dependent population dynamics, Arch. Rat. Mech. Anal. 82 (1983) 13-25. 